\title{
RESEARCH
}

Open Access

\section{CooC11 and CooC7: the development and validation of age appropriate children's perceived cooking competence measures}

Moira Dean ${ }^{1,2}$, Johann Issartel ${ }^{3}$, Tony Benson ${ }^{1}$, Amanda McCloat $^{4}$, Elaine Mooney ${ }^{4}$, Claire McKernan ${ }^{1}$, Laura Dunne ${ }^{5}$, Sarah F. Brennan ${ }^{1,6}$, Sarah E. Moore ${ }^{1,6}$, Danielle McCarthy ${ }^{1}$, Jayne V. Woodside ${ }^{1,5,6}$ and Fiona Lavelle 1* $^{*}$

\begin{abstract}
Background: Learning cooking skills during childhood and adolescence is associated with positive dietary outcomes in adulthood as well as being tracked from adolescence to adulthood. In addition studies have found that perceived competence to be a greater motivator to perform a behaviour than actual competence. However, a lack of validated tools that effectively measure behavioural and dietary changes including cooking confidence in children is a limitation. Therefore, this research aimed to develop and validate age-appropriate perceived cooking competence measures for younger and older primary school aged children.

Methods: Two measures of perceived Cooking Competence (CooC11 and CooC7) for older (8-12 years) and younger (6-7 years) children were developed from a critical evaluation of publically available recommendations and expert consultation. The cooking skills within the measures were illustrated by a graphic designer in consultation with a chef and reviewed in an iterative manner by the research team. The measures were piloted for clarity, ease of use and initial face validity. Multiple studies were used for both $\mathrm{CooC11}$ and $\mathrm{CooC7}$ to establish psychometric properties of the measures, temporal stability, internal consistency reliability, construct validity, as well as responsiveness to change for CooC11. Analysis included Exploratory Factor Analysis, Confirmatory Factor Analysis, Intraclass Correlation Coefficients, Pearson's Correlations, ANOVAs and Cronbach's Alphas.

Results: Both measures had high levels of face validity and received positive user feedback. Two factors were shown in both measures with the measures showing excellent temporal stability (ICC $>0.9$ ) and good internal consistency (Cronbach's Alphas $>0.7$ ). Both measures showed initial discriminant validity, with significant differences $(P<0.001)$ between those who reported assisting their parents with dinner preparation and those who did not. Additionally, CooC11 was significantly correlated with an adult cooking measure and had a significant responsiveness to change $(P<0.01)$.

(Continued on next page)
\end{abstract}

\footnotetext{
* Correspondence: flavelle01@qub.ac.uk

'Institute for Global Food Security, School of Biological Sciences, Queen's University Belfast, Belfast, UK

Full list of author information is available at the end of the article
}

(c) The Author(s). 2021 Open Access This article is licensed under a Creative Commons Attribution 4.0 International License, which permits use, sharing, adaptation, distribution and reproduction in any medium or format, as long as you give appropriate credit to the original author(s) and the source, provide a link to the Creative Commons licence, and indicate if changes were made. The images or other third party material in this article are included in the article's Creative Commons licence, unless indicated otherwise in a credit line to the material. If material is not included in the article's Creative Commons licence and your intended use is not permitted by statutory regulation or exceeds the permitted use, you will need to obtain permission directly from the copyright holder. To view a copy of this licence, visit http://creativecommons.org/licenses/by/4.0/ The Creative Commons Public Domain Dedication waiver (http://creativecommons.org/publicdomain/zero/1.0/) applies to the data made available in this article, unless otherwise stated in a credit line to the data. 
(Continued from previous page)

Conclusions: The $\mathrm{Coo} \mathrm{C} 11$ and $\mathrm{CooC} 7$ are the first validated age-appropriate measures for assessing children's perceived Cooking Competence for ages 8-12 and 6-7 years respectively. They can be used to evaluate the efficacy of children's cooking intervention studies or school nutrition education programmes.

Keywords: Measure, Development, Validation, Cooking, Children, Intervention, Assessment, Nutrition, Motor skills, Competence

\section{Background}

Diet quality has been associated with a number of health outcomes including all-cause mortality, cardiovascular disease risk, cancer risk, and obesity, as well as nonhealth related outcomes such as academic performance [1-4]. Due to the beneficial associations of a higher diet quality, nutrition education programmes are being used for changing children's dietary patterns and intakes to increase healthy eating practices $[5,6]$. Within this area, cooking interventions have been highlighted as a promising method for changing children's food-related attitudes, preferences and behaviours $[7,8]$. Research shows that learning cooking skills at younger ages is associated with positive dietary outcomes in adulthood and these skills track from adolescence to adulthood $[9,10]$. Additionally, consumption of meals prepared in the home environment, which require cooking skills, has been associated with a normal BMI and body fat percentage [11]. However, a lack of validated measurement tools that effectively measure behavioural and dietary competencies in children is a limitation not only in cooking research [8] but is also an issue in the wider nutrition area [12, 13].

In recent years, a small number of child-orientated measures have been developed in the nutrition area covering topics such as Nutrition and Food Label Literacy $[14,15]$. Yet, parental perspectives of child behaviours are still often used as a measure which can lead to bias [16]. Within children's cooking interventions, while some effort has been made to develop a validated measure [17], this measure tends to focus on broader concepts such as preparing a snack with fruit or vegetables, following a recipe or making a salad etc. and are not specific to measuring individual cooking skills such as chopping, stirring or peeling.

In both adults and children, increased confidence and self-efficacy (situation specific self-confidence [18]) are key contributors to engaging in cooking practices and repeating the behaviour [19-23]. The self-efficacy people have for a specific task, in this case cooking, contributes to the individual's perceived competence ('the perception a person has concerning his or her abilities' [24]). Studies have found perceived competence to be a greater motivator to perform a behaviour than actual competence [25]. This has been extensively studied in the area of physical activity, where children with higher levels of perceived competence participated in a greater amount of physical activity [26]. Additionally, higher levels of perceived competence at younger ages predicted higher levels of perceived competence and physical activity at older ages [27]. Furthermore, children with low levels of perceived competence, even with high actual competence, were shown to have lower levels of motivation for physical activity than children with high levels of perceived competence (with or without matching levels of actual competence) [28]. Therefore, being able to measure perceived competence effectively is essential for understanding behaviour change and for evaluating successful interventions. While measuring perceived competence in motor skills exist $[29,30]$ currently there is no equivalent perceived competence measurement tool in the area of cooking, which is also a learned and modifiable behaviour.

The developmental differences between children and adults require consideration when conducting research with children [31]. Therefore, when developing measures appropriate to children, the developmental stages and capabilities of the child, such as their motor skill development, must be taken into consideration for the content of the measure, as well as a child's attention span, the format of the measure, the validity and reliability of the responses and the clarity of the language [31, 32]. In addition, recommendations from the literature [31, 33] suggest that, when children are involved, research methods using visual and/or game-like measurements are preferred. These methods are more engaging to children and are similar in formats to teaching methods used at school $[31,33]$. These strategies have been implemented in the children's perceived motor skills competence measure $[29,30]$. They have shown to be effective in the measurement of perceived motor skills competence, globally [34-38]. However, while using these methods may be engaging for the children, it must also obtain relevant data [31]. Therefore, it is necessary to develop appropriate and relevant items within the measure. The specific cooking skills within the measure must be relevant to the children's developmental capacity to ensure that children are rating their perceived competence on items that they are able to achieve (i.e. appropriate for their age) [39]. Therefore, this research 
aimed to develop and validate age-appropriate perceived cooking competence measures for children, through the assessment of the measures' face validity, psychometric properties, construct validity and reliability (both internal consistency and temporal stability).

\section{Methods}

A number of steps were undertaken in the development and validation processes of the two age-appropriate children's cooking competence measures. Items were selected for inclusion for the content of the measures from a review of children's cooking recommendations that were mapped to their age-appropriate developmental skills [39], and were reviewed by an expert panel. The measure was designed in line with a published perceived motor competence measure [30] and characters performing the cooking skills were illustrated by a graphic designer. Next initial face validity was established by the research team and a primary school teacher and the measures were piloted. The psychometric properties of the measures were assessed using Exploratory Factor Analysis (EFA) and Confirmatory Factor Analysis (CFA) and face validity of the final structures of the two measures were established. Following this, construct validity, convergent and discriminant, and internal consistency reliability were assessed. Temporal stability reliability of both measures was then examined. Finally, for the older measure, responsiveness to change was established. The details of this process can be found in the following sections.

\section{Item selection}

The cooking skills that children should be learning at different ages were obtained through a critical evaluation of publically available children's recommendations and the addition of new recommendations based on children's developmental skills [39]. From this review, for the two Cooking Competence measures, 14 cooking skills for 8-12 year olds (CooC11) and 10 skills for 6-7 year olds (CooC7) were identified as being frequently occurring and culturally neutral, i.e. a cooking skill was not specific to one culture. The items were selected to ensure they were both developmentally appropriate and relevant [31]. An expert panel including an educational researcher, a primary school teacher, an early year's educator, a movement scientist and two Home Economists, with a minimum of 10 years' experience in their respective fields, reviewed the selected skills for age appropriateness and level of difficulty. An age range was proposed for each skill and skills were then ranked in order of difficulty from easiest to hardest, see Table 1.

\section{Development and implementation of measure}

The design of the children's perceived cooking competence measure was based on a published perceived motor competence measure [30]. However, in the

Table 1 Cooking skills identified for younger and older children

\begin{tabular}{|c|c|c|}
\hline Level of difficulty & Cooking Skills & \\
\hline & Younger (ages 6-7years) & Older (ages 8-12 years) \\
\hline \multicolumn{3}{|l|}{ Easiest } \\
\hline & Tearing Leaves & Tearing Leaves \\
\hline & Washing Vegetables & Washing Vegetables \\
\hline & Stirring/Mixing ingredients & Stirring/Mixing ingredients \\
\hline & Mashing & Mashing \\
\hline & Measuring liquids ${ }^{a}$ & Measuring liquids ${ }^{a}$ \\
\hline & Weighing ingredients ${ }^{a}$ & Weighing ingredients ${ }^{\mathrm{a}}$ \\
\hline & Chopping & Chopping \\
\hline & Grating & Using a blender ${ }^{c}$ \\
\hline & Peeling & Grating \\
\hline & Using a tin opener ${ }^{\mathrm{b}}$ & Peeling \\
\hline & & Using a microwave ${ }^{d}$ \\
\hline & & Using a tin opener \\
\hline & & Using the oven ${ }^{d}$ \\
\hline Most difficult & & Using the stove/hobe \\
\hline
\end{tabular}

a - measuring liquids and weighing were separated in the measure; ${ }^{b}$ - tin opener was placed in the younger age before the expert panel review moved it into the $9+$ age category; ${ }^{c}$ - blender replaced mixer as mixer was more associated with baking as opposed to cooking; ${ }^{d}$ - oven and microwave were separated in the measure; ${ }^{\mathrm{e}}$ - Using the stove/hob was added as a means of factoring in the use of a cooker/cooker top for those that may not have an oven/as a means of trying to include stirring over heat. 
cooking skills measure the child was asked first whether they engaged in a particular cooking skill (in line with Lavelle et al. [40]), before they rated their level of competence. This aims to reduce positive illusory and social desirability biases [31]. In the measure, each skill was illustrated as a child-friendly character performing the skill. Using an iterative process, the child characters were drawn by a graphic designer in consultation with a chef and reviewed by the research team for accuracy and suitability. The illustrations provided a visual 'cue' to the cooking skill as some cooking terminology relating to skills may not be familiar to the children. In line with Barnett et al. [30], the child is shown an image of a child, boys are presented with images of boys performing the skills and girls are shown images of girls. This promotes a peer modelling effect, as it is argued that a child is more likely to relate to a character that is more like themselves [41, 42]. The child is asked whether they do the skill shown (see Fig. 1 as an example). If the child responds yes, then they are shown two more images of the child performing the skill, one performing it well and the other performing it poorly. The child is then asked which image represents their perceived level of competence on a five point Likert scale.

The five response options result in five possible levels of competence for each skill (see Fig. 2 as an example). However if the child responds that they do not perform that particular skill, they move on to the next skill.

The cooking skills are presented in ascending level of difficulty as rated by the expert panel. Additionally, the

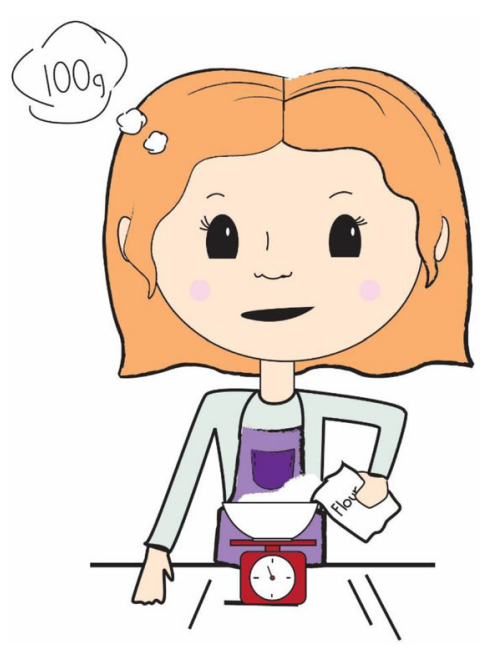

This child is weighing ingredients. Do you do this?

$$
\begin{array}{ll}
\text { O Yes } \\
\text { O No }
\end{array}
$$

Fig. 1 Exemplar of a cooking skill (female version) sequence of presentation of 'good' competence of a skill alternated in position on the page with 'poor' competence of a skill [29, 30]. Each child completes the measure individually. However, if a child's literacy levels were not at a sufficient level that they could read the questions, then the researchers assisted the child by reading out the questions so that the child could complete the task.

\section{Piloting and initial face validity}

The measures were reviewed by the research team and a primary school teacher for language, readability and literacy levels [31]. Based on the feedback minor amendments were made to the language, such as changing in between A and B' to 'A bit like A and B,' as it was suggested that children would interpret the original phrasing to mean physically in between the two characters. Additionally, the font size of the text was increased for the younger age group. Furthermore, the characters' expressions were all changed to neutral, so that the children would not choose their responses based on how happy or sad they were feeling but on their perceived level of competence. Thus, the research team assessed the measures for initial face validity.

The designed measures were also piloted with a number of children of differing ages [43]. This piloting allowed the research team to assess the usability, length of time of completion, enjoyability as well as further face validity such as recognition of the skills and differentiation between the 'good,' and 'poor' performance of the skill.

Further validation assessments were undertaken for both the older age measure (CooC11) and the younger age measure (CooC7), which will be detailed in the following section. For both measures, endpoint user feedback from both the children and teachers were received. Five teachers and three teaching assistants, from the recruited samples of children, provided their perceptions around the measures including the suitability, usability and length. Each class in these samples were asked about their experiences using the measure, whether they liked this type of activity, about the characters and if there was anything they would change. Additionally, informal qualitative feedback was gathered across all samples. Prior to data analysis, where necessary, items were reversed coded so that a higher score indicated greater perceived cooking competence for all items. All analyses were conducted using IBM SPSS Statistics v25 and IBM SPSS Amos v25, with a significance level of 0.05.

\section{CooC11 (8-12 year olds) Participants and procedure}

Sample 1: Data from 469 primary school children aged 10-11 years completed baseline measurements as part of 


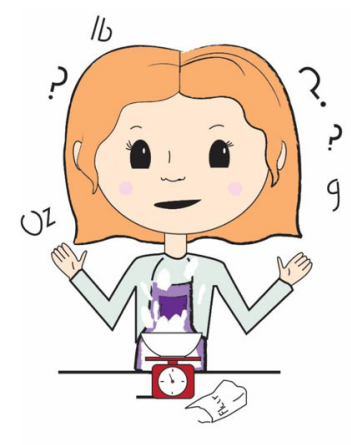

A is not that good at weighing

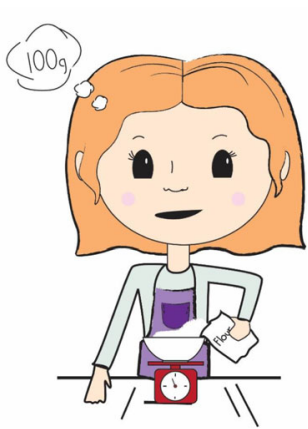

$B$ is really good at weighing

Which are you MOST like?
- I am a lot like $A$
- I am a little like A
- I am a bit like A and $\mathrm{B}$
- I am a little like B
- I am a lot like B

Fig. 2 Exemplar of poor and good performance of a cooking skill presented to a child if they have indicated that they perform the skill

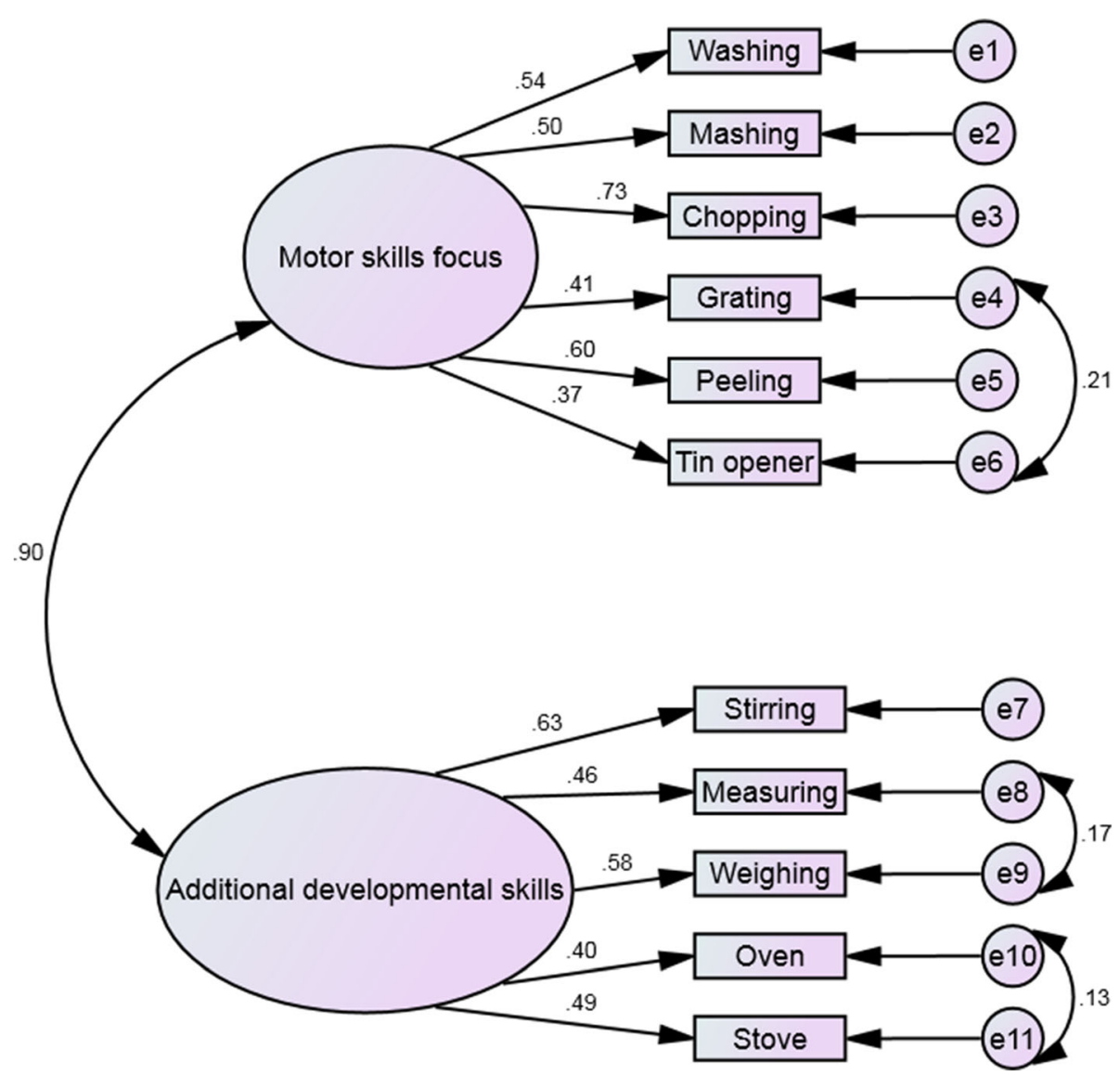

Fig. 3 Final measurement model for $\mathrm{CooC} 11$ with standardised factor loadings and correlations 
a larger study (Project Daire) [44]. For this sample, $50.32 \%$ were female. Schools from both rural and urban areas with varying socioeconomic levels were included. Data was collected in February-March 2019.

Sample 2: Children $(N=38)$ between the ages of $8-9$ years and 10-11 years (two year groups in the primary school system in Northern Ireland) were recruited. Children from one primary school were recruited for this study. For this sample, 52.6\% were female. Children in this sample completed the measure at two time points two weeks apart in May 2019.

Sample 3: Children $(N=32)$ between the ages of $10-12$ years who participated in a one week cooking camp intervention in August 2019. In this sample, 78.1\% were female. These children completed the measure before and after the cooking camp intervention.

\section{Psychometric testing, validation and data analysis}

Exploratory factor analysis Sample 1 was randomly split to conduct EFA and CFA, with 269 children included in the EFA. EFA (maximum likelihood) with direct oblimin rotation was used. This oblique rotation was used as it was believed that factors would be related [45]. Sample adequacy was assessed using Kaiser-MeyerOlkin (KMO) value [46] and Bartlett's Test of Sphercity [47]. Factors were assessed using Eigenvalues greater than 1 [48] and a minimum of 3 items per factor [49]. Items were removed based on communalities and factor loadings.

Confirmatory factor analysis and face validity The remaining 200 randomly selected children from sample 1 were used for CFA. The final model identified by the EFA was assessed as a confirmatory factor analysis with maximum-likelihood estimation, using IBM SPSS Amos v25. The following fit statistics were used to assess the model [50]:

- Chi-square $\left(\mathrm{x}^{2}\right)$ - A non-significant chi-square value $(p>0.05)$ which is two or three times larger than its value divided by the degrees of freedom (df) at its maximum indicates that the model can be accepted.

- Root Mean Square Error of Approximation (RMSE A) - A preferred value is 0.05 or less.

- Comparative Fit Index (CFI), Normed-Fit Index (NFI), Tucker-Lewis Index (TLI) - For these indices a value of 0.90 or greater indicates that the model can be accepted.

To establish face validity of the measure structure, five researchers in the areas of food, nutrition, health psychology, Home Economics and human movement science, reviewed the final model and factor structure. Cooking skills in each factor were assessed upon general relation in cooking as well as underlying developmental skills including fine and gross motor skills in addition to numeracy, literacy and safety considerations. All items were assessed to ensure they measured what they claimed to measure.

Construct validity - convergent and discriminant validity Sample 1 was used for Construct validity. Convergent validity shows that measures are valid by identifying a relationship with an existing similar measure using correlation analysis. As there are no similar children's measures to establish convergent validity, the cooking method section of an adult measure was used [40]. This measure has not been used previously with children due to the levels of literacy required. However, as the current sample is at the older end of the age range for the measure, the research team decided to include the measure as a means of establishing some level of convergent validity. Additionally, due to the lack of measurements available, the children were asked whether they help their parents making the dinner. It was expected that those who assist with dinner preparation would have a reported higher cooking competence. Due to a large number of children answering 'sometimes' $(N=298)$ and a small number answering 'always' $(N=35)$, compared with those answering 'never', 'sometimes' and 'always' were combined to those who 'help with dinner.' And due to the large difference between the two response categories, a random selection of those who responded 'help with dinner' were selected to compare against never. This ensured that there was a relatively equal number of participants in each group for the oneway ANOVA.

Internal consistency reliability Internal consistency reliability was used to examine agreement between the items in a scale. Cronbach's Alpha was used to assess internal consistency reliability. A value of 0.7 or higher shows good reliability [51]. Sample 1 and 2 were used to establish internal consistency of the measure.

Temporal stability Sample 2 was used to assess Temporal Stability of the measure. The temporal stability of the scales was examined using the Intraclass Correlation Coefficient (ICC). This illustrates the level of agreement between item answers over time. A stronger ICC indicates greater agreement, suggesting greater temporal stability. Moderate reliability is seen with an ICC value of $0.50-0.75$, good reliability is a value of 0.75 0.90 , while a value of greater than 0.90 suggests excellent reliability [52]. 
Responsiveness to change Sample 3 was used to assess the responsiveness to change of the measure, a further indication of validation [53]. This was established through investigating changes in the measure scores before and after the children receive a cooking focused intervention using T-tests.

\section{CooC7 (6-7 year olds)}

\section{Participants and procedure}

Sample 4: Data from 514 primary school children aged 6-7 completed baseline measurements as part of a larger study (Project Daire) [44], are used as Sample 4. For this sample, $48.63 \%$ were female. Schools with varying socioeconomic levels and from both rural and urban areas were included. Data was collected in FebruaryMarch 2019.

Sample 5: Children $(N=13)$ between the ages of $6-7$ years old were recruited as part of Sample 5 from the same school as sample 2 . In this sample, $46.2 \%$ were female. Children in this sample completed the measure at two time points two weeks apart in May 2019.

\section{Psychometric testing, validation and data analysis}

The same criteria as in 2.4.2 were used for testing the $\mathrm{CooC7}$ measure. The samples used, and differences in analysis to CooC11 are detailed below.

Exploratory factor analysis Sample 4 was randomly split to conduct Exploratory Factor Analysis (EFA) and Confirmatory Factor Analysis (CFA), with 314 children included in the EFA.

Confirmatory factor analysis and face validity The remaining 200 randomly selected children from Sample 4 were used for CFA. The same procedure was used for the CFA and face validity as in section 2.4.2.

Construct validity - discriminant validity Sample 4 was used for construct validity. There are no similar children's measures to establish convergent validity and the cooking method section of the adult measure [40] is above the literacy and cognitive capacity of this age group. Due to the lack of measurements available, the children were asked whether they help their parents making the dinner, with the expectation again that a higher cooking competence score would be seen in those that help with dinner preparation. The responses for the children were 'never', 'sometimes' or 'always'. Comparisons between children in the 3 categories were conducted using an ANOVA with Scheffe post hoc analysis due to differences in numbers in the groups.

Internal consistency reliability and temporal stability Sample 4 and 5 were used to establish internal consistency of the measure. Sample 5 was used to assess Temporal Stability of the CooC7.

\section{Ethical considerations}

All schools partaking in the research (Samples 1, 2, 4 and 5) signed and returned a memorandum of understanding. An opt-out parental consent system was implemented. In sample 3 , due to the nature of the intervention and the demand for places, an opt-in system was used. In all samples, parents were made aware that they were not obliged to allow their child to take part in the study and that they could withdraw their child at any time point up to data analysis without reason or consequence. Additionally, the children were made aware that they did not have to take part. The research was conducted in accordance with the Declaration of Helsinki. Ethical approval was received from The School of Social Sciences, Education and Social Work Ethics Committee, Queen's University Belfast (Reference number 038_1819) for Samples 1 and 4 and from The School of Biological Sciences Ethics Committee, Queen's University Belfast (0519/LavelleFA, 0519/ LavelleFB), for Samples 2, 3 and 5.

\section{Results}

\section{Overall usability, face validity, user feedback}

The research team established initial face validity to ensure that all items measured what they claimed to measure. Piloting of the measures established that children could distinguish between 'good' and 'poor' performance of an illustrated skill and that they found the measure easy to use. Teacher feedback relating to the characters was positive and the teachers felt that the illustration would help the children struggling with literacy and/or would help children who had learning difficulties. However, it was noted that some children using the $\mathrm{CooC7}$ may still need help reading. Teachers recommended that a larger font size for CooC11, would be beneficial for the younger age group (8-9 years) to help with their reading. The children's enjoyment completing the measure, especially using the measure on a tablet, and the short duration of time required to complete the measures were seen as positives.

Qualitative feedback showed that children completing the measure enjoyed doing it and wanted more questions and suggested that they should be given an opportunity to provide a reason why they don't do certain skills. They also identified with the illustrated characters, "That guy is just like me except the hair - like it's me" (P7 male pupil, sample 1).

\section{CooC11 (ages 8-12 years) \\ Exploratory factor analysis}

The results showed an excellent KMO value of 0.86 and a significant $(p<.001)$ Bartlett's Test of Sphericity, 
indicating sample adequacy for analysis. Initially three factors were apparent in the data. 'Tearing leaves,' 'Using a blender,' and 'Using a microwave' were removed due to communalities $<0.25$ and cross loading across factors. Given this the analysis was conducted again, to ensure that the factor structure and results were acceptable after the modification. Two factors were now apparent in the data, as shown by Eigenvalues greater than 1. Both factors had a minimum of 3 items, no items crossloaded on more than one factor, and the minimum factor loading was 0.3 . In addition, the internal reliability values for each factor were 0.77 and 0.72 respectively, therefore, all 11 items were retained. The overall Cronbach's alpha for the measure was 0.82 in this sample.

\section{Confirmatory factor analysis}

When entered as a CFA, the final EFA model did not have optimal fit (significant $\chi^{2}$, RMSEA 0.06, NFI 0.83, TLI .89). To improve this, the modification indices suggested some of the error terms should be allowed to covary. Following these amendments, fit was acceptable. Specifically, the $\chi^{2}$ was significant, but with a $\chi^{2} / \mathrm{df}$ ratio below 2 (1.50). RMSEA was 0.05 . While the NFI was .87 , the CFI was .95 and TLI .93, indicating overall acceptable fit. All standardised loadings were 30 or above (see Fig. 3 for final model). Face validity was established through the agreement that all items were appropriate for their factor, after discussion around 'stirring.' As 'stirring' may seem more appropriate to a motor skills focus, however, as highlighted in the original review, 'stirring' could be considered over heat, which has additional safety components. Due to it being considered an easier skill, it appeared before 'using a hob/stove' which was added to factor in this difference and therefore some children may have still considered it over heat, and thus agreement was reached it was appropriately placed.

\section{Construct validity (convergent and discriminant validity)}

In sample $1(N=469)$, the adults cooking methods confidence score had a Cronbach's Alpha of 0.82. Spearman's rank correlation analyses showed that the children's cooking competence measure in the current study was significantly correlated with the adults cooking methods confidence score, $0.49(P<0.001)$. Those children that reported helping their parents with preparing the dinner had a significantly higher cooking competence than those who did not $(\mathrm{P}<0.001)$, see Table 2 .
Table 3 Temporal Stability of CooC11 and factors

\begin{tabular}{llll}
\hline Scale & T1 & T2 & ICC \\
\hline & M (SD) & M (SD) & \\
Motor skills focus & $7.50(8.04)$ & $6.97(7.35)$ & 0.89 \\
Additional Developmental skills & $6.37(6.44)$ & $7.61(7.11)$ & 0.85 \\
CooC11 & $\mathbf{1 3 . 8 7 ( 1 3 . 4 2 )}$ & $\mathbf{1 4 . 5 8 ( 1 3 . 1 6 )}$ & $\mathbf{0 . 9 1}$ \\
\hline
\end{tabular}

\section{Internal consistency and temporal stability}

The internal consistency reliability of CooC11 was very good for the 8-9 years and 10-11 years, with a Cronbach's of 0.86 and 0.84 , respectively, and 0.85 overall.

In terms of temporal reliability CooC11 had an ICC of 0.91, indicating an excellent temporal stability, with the two subscales having good temporal stability, as detailed in Table 3.

\section{Responsiveness to change}

The measure is responsive to change, as seen by a significant increase $(P<0.01)$ from pre-cooking camp intervention CooC11 mean (SD), 21.75 (7.89), to post camp CooC11, 26.13 (8.89).

\section{CooC7 (ages 6-7 years) \\ Exploratory factor analysis}

The results showed an excellent KMO value for CooC7 of 0.81 and a significant $(\mathrm{p}<.001)$ Bartlett's Test of Sphericity, indicating that the sample was adequate for factor analysis. Two factors were seen in the data, as shown by Eigenvalues greater than 1. All factors had a minimum of 3 items. 'Tearing leaves' and 'Stirring/mixing' were removed due to communalities $<0.15$. Furthermore, 'Using a tin opener' was removed as it did not meet the minimum factor loading of 0.3 and cross loaded across factors. Given this the analysis was conducted again to ensure that the factor structure and results were acceptable following the previous modification. All factors contained at least three items, no items cross-loaded on more than one factor, and the minimum factor loading was 0.3. In addition, the Cronbach's Alpha values for each factor were 0.65 and 0.62 , respectively, therefore, all 7 items were retained. The overall measure had an internal consistency reliability of 0.71 .

\section{Confirmatory factor analysis}

The final EFA model fit the data well in the confirmatory model (Fig. 4). The $x^{2}$ was non-significant, with a

Table 2 Differentiating between those that report helping prepare dinner and those that don't

\begin{tabular}{lllll}
\hline Measure & Total Sample $(\boldsymbol{N}=\mathbf{2 7 2})$ & Do not help with dinner $(\boldsymbol{N = 1 3 6 )}$ & Help with dinner $(\boldsymbol{N = 1 3 6})$ & Significance \\
\hline & $\mathrm{M}(\mathrm{SD})$ & $\mathrm{M}(\mathrm{SD})$ & $\mathrm{M}(\mathrm{SD})$ & $\mathrm{P}$ \\
CooC11 & $17.04(12.88)$ & $10.57(9.65)$ & $23.51(12.47)$ & 0.000 \\
\hline
\end{tabular}




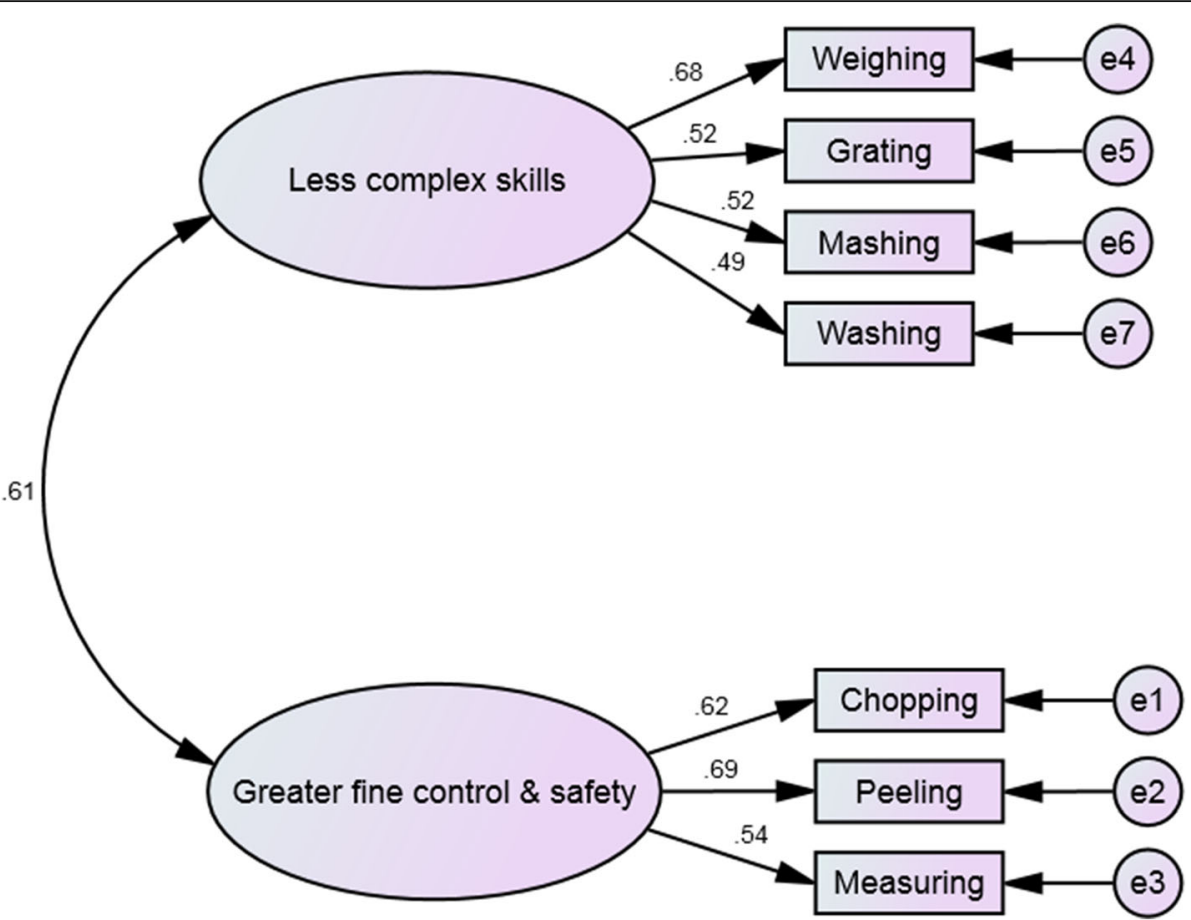

Fig. 4 Final measurement model for $\mathrm{CoOC7}$ with standardised factor loadings and correlations

$\mathrm{X}^{2} / \mathrm{df}$ ratio of 1.24 , and the RMSEA was 0.04 . The CFI, NFI, and TLI were all excellent at .98, .93, and .98 respectively. In addition, all standardised loadings were above 3 . Face validity was established through the agreement that all items were appropriate for their factor after discussion around the factoring of weighing and measuring on separate factors.

\section{Construct validity (discriminant validity)}

Discriminant validity results for $\mathrm{CooC7}$ can be seen in Table 4 below.

\section{Temporal stability}

In terms of temporal reliability the measure had an ICC of 0.92, indicating an excellent temporal stability, with the two subscales having good temporal stability (see Table 5).

\section{Discussion}

To the best of our knowledge, this research is the first to develop and validate age appropriate children's measures, CooC11 and CooC7, for the design and evaluation of nutrition and cooking interventions. Effectively measuring perceived cooking competence is an important element for assessment of interventions, as perceived competence has been shown to be a motivator for repeating the behaviour [25] and cooking has been associated with a better diet quality $[7,8]$. Both measures were developed to ensure they were age appropriate and engaging for children [31,33]. They have high levels of face and construct validity and excellent internal consistency reliability and temporal stability. Additionally, CooC11 demonstrated responsiveness to change validity.

For both measures endpoint user feedback was generally positive and children found completing the measures enjoyable. The children understood the language being used, enjoyed the character illustrations and use of a tablet to complete the measure, indicating an appropriate measure format, and requested additional questions, highlighting that completing the measure was within their attention span [31, 32].

Table 4 Differentiating between the different levels of assisting preparing dinner

\begin{tabular}{llllll}
\hline Measure & $\begin{array}{l}\text { Total Sample } \\
(\boldsymbol{N}=\mathbf{5 1 3})\end{array}$ & $\begin{array}{l}\text { Never help with } \\
\text { dinner }(\boldsymbol{N = 1 9 0 )}\end{array}$ & $\begin{array}{l}\text { Sometimes help with } \\
\text { dinner }(\boldsymbol{N}=\mathbf{2 0 5})\end{array}$ & $\begin{array}{l}\text { Always Help with } \\
\text { dinner }(\boldsymbol{N}=\mathbf{1 1 8})\end{array}$ & $\begin{array}{c}\text { Significance } \\
\text { CooC7 }\end{array}$ \\
\hline & $M(S D)$ & $M(S D)$ & $M(S D)$ & $M(S D)$ & $P$ \\
\hline
\end{tabular}

Superscript letters depict where significant differences $(P<0.001)$ fall between the groups. 
Table 5 Temporal Stability of measure and factors

\begin{tabular}{llll}
\hline Scale & T1 & T2 & ICC \\
\hline & $M(S D)$ & $M(S D)$ & \\
Less Complex skills & $7.00(4.81)$ & $7.62(7.07)$ & 0.85 \\
Greater fine control \& Safety & $5.23(5.00)$ & $4.77(4.89)$ & 0.86 \\
CooC7 & $\mathbf{1 2 . 2 3 ( 9 . 0 0 )}$ & $\mathbf{1 2 . 3 9 ( 1 0 . 1 4 )}$ & $\mathbf{0 . 9 2}$ \\
\hline
\end{tabular}

\section{Factors}

The EFA and CFA confirmed a two-factor structure for both CooC11 and CooC7. For CooC11, face validity of the two factors was established, Factor 1 consists of cooking skills that encompass basic motor skills and Factor 2 consists of cooking skills that need additional developmental skills such as numeracy and literacy. Stirring was accepted in Factor 2, as this cooking skill is often performed over heat and therefore needed safety considerations, which was also noted in the original recommendations [39]. While the skill 'using a stove/hob' was added to encompass this type of stirring, e.g. stirring over heat, the skill 'stirring' is shown to the child before 'using a stove/hob' so they may still consider 'stirring' as over heat.

CooC7, also has two factors. Factor 1 consisted of the less complex skills for this age group whereas, Factor 2 consisted of cooking skills that required greater fine motor control and safety aspects. While there was some initial discussion around 'grating' factoring onto Factor 1 , it was established that this cooking skill requires less fine motor control than 'using a peeler' or 'chopping.' Additionally, in this age group, 'measuring liquids' and 'weighing' factored onto different factors. It is suggested that for this age group, 'measuring liquids' is a more complex skill requiring greater fine motor control to accurately measure out liquids correctly.

While these factors are apparent within the measures, using CooC11 and $\mathrm{CooC7}$ in their entirety is currently recommended, as these measure are quick to complete and there is a lack of validated measures to assess the factor construct validity.

\section{Validity and reliability}

The CooC11, was correlated with an adult cooking confidence measure [40], which showed some convergent validity. Both measures were able to distinguish between children who reported different levels of assisting their parents with preparing the dinner, highlighting initial discriminant validity. Furthermore, CooC11 showed it was responsive to change, as there was a significant increase in perceived cooking competence by children attending the cooking camp intervention. It is worth noting that due to the nature and recruitment for this intervention, these children had an initial interest in cooking, shown by their higher initial CooC11 scores compared to the children in samples 1 and 2 who were recruited from schools. As changes were detected pre and post intervention, this demonstrates that the measures can be used with participants who have some level of initial cooking competence to measure changes.

Both measures showed that they had high levels of internal consistency reliability, indicating that they are measuring coherent concepts, demonstrated by the Cronbach's alphas being greater than 0.7, which is satisfactory for non-clinical samples [54, 55]. Additionally, both overall measures showed excellent temporal stability demonstrated by the test-retest analysis, where the ICC scores were greater than 0.9 [52], showing that the scores are highly reliable over time.

\section{Strengthening the research area}

The reported decline in child involvement in cooking in the home environment $[56,57]$, has led to an increase in children's cooking interventions [7, 8]. New models are being created, such as the Cook-Ed ${ }^{\text {TM }}$ model [58] to help guide the design, development and evaluation of the quality and success of the interventions using validated measures. The new CooC11 and $\mathrm{CooC7}$ are a necessity in this area and will contribute to the strengthening of the global research by providing validated measurements to use in the evaluation of intervention studies. The measurement of perceived competence is a key element for consideration in the evaluation of interventions, as perceived competence has been shown to be a motivator for repeating the behaviour [25].

\section{Future research}

Future research should assess the responsiveness to change of CooC7. Due to the difficulty in recruiting this age group within the available resources this was not assessed within the reported studies. Endpoint user feedback from teachers suggest that these new measures can be adapted to be used with people with learning difficulties and/or disabilities. Future research could develop and diversify the characters to make the measures suitable to use with these target populations.

\section{Strengths and limitations}

The new measures involved extensive development, underpinned by a review, expert consultation and existing measures $[29,40]$ and illustrated by a graphic designer in consultation with a chef. The illustrated characters were highlighted as helpful and relatable. Additionally, the use of tablets to complete the measure was enjoyable to the participating children and the use of characters and a tablet to undertake the measures, enabled a more 'game' feeling rather than a test, which was found to be beneficial [33]. However, it was noted, to help with literacy for those at the younger end of the age 
range using CooC11, an increased font size would be beneficial.

The developed measures were found to be highly reliable and valid. The cooking skills included were based on evidence based age-appropriate recommendations developed from global publically available cooking recommendations and deconstructed for their underlying motor skills [39], thus increasing the generalisability of the measures outside of a UK/Irish population. Due to the lack of ethnic diversity in the regions being sampled, the children were shown Caucasian illustrations, however, to increase the cross-cultural applicability of the measures, a range of diverse character illustrations are available to use to ensure that all children can identify with the characters illustrated. While the measures assess specific skills and the illustrations only act as a 'prompt' for skill identification, some examination of the use of different characters in the measure may be of interest. Furthermore, if individual factors in the measures are to be used as standalone measures then construct validity of the factor structure is necessary when additional measures are available.

\section{Conclusions}

The $\mathrm{CooC11}$ and $\mathrm{CooC7}$ are the first extensively developed and validated age-appropriate measures for assessing children in nutrition related interventions. The measures assess children's perceived Cooking Competence for children aged 8-12 and 6-7 years respectively and can be used to evaluate the efficacy of children's cooking intervention studies or school programmes.

\section{Acknowledgements}

The authors would like to thank the pupils and their parents, principals, teachers from all the schools, from the piloting phase and from the 'Cook Like A Boss' cooking intervention that participated in the research. Additionally, the authors are grateful for the input from the experts involved in the initial review of the items. The authors would like to thank Lucy Patterson (@lucy_graphicdesigner) for her design work.

\section{Authors' contributions}

FL created the initial concept with development and research design contributions from MD and $\mathrm{Jl}$. JI, AMC, EM and LD were involved in the expert review. JWW, CMC, DMC, SFB, SEM and FL were involved in recruitment and data collection. FL and TB conducted the majority of the analysis with input from JI, AMC and EM. FL and MD drafted the manuscript. All authors reviewed and edited the manuscript. The authors read and approved the final manuscript.

\section{Funding}

Funding for character illustrations through Project Daire was received from the Agri-Food Quest Competence Centre, funded by Invest Northern Ireland. The study funder had no role in the study design, collection, analysis or interpretation of the data and had no role in writing the manuscript.

\section{Availability of data and materials}

The datasets used and/or analysed during the current study are available from the corresponding author on reasonable request. The measures are freely available to use and can be obtained by contacting the corresponding author. Additionally, the measure has been translated into the Irish language which is also available from the corresponding author. Where appropriate the corresponding author is willing to assist with back translation for other translations.

\section{Ethics approval and consent to participate}

Ethical approval was received from The School of Social Sciences, Education and Social Work Ethics Committee, Queen's University Belfast (Reference number 038_1819) for Samples 1 and 4 and from The School of Biological Sciences Ethics Committee, Queen's University Belfast (0519/LavelleFA, 0519/ LavelleFB), for Samples 2, 3 and 5. Informed parental consent was received from all parents through an opt-out system in all schools samples and an opt in system in Sample 3 due to the nature of the intervention. In all samples, parents were made aware that they were not obliged to allow their child to take part in the study and that they could withdraw their child at any time point up to data analysis without reason or consequence.

\section{Consent for publication}

Not applicable.

\section{Competing interests}

The authors declare that they have no competing interests.

\section{Author details}

${ }^{1}$ Institute for Global Food Security, School of Biological Sciences, Queen's University Belfast, Belfast, UK. ${ }^{2}$ School of Health Sciences, Faculty of Health and Medicine, The University of Newcastle, Callaghan, NSW 2308, Australia. ${ }^{3}$ Multisensory Motor Learning Lab, Dublin City University, Dublin, Ireland. ${ }^{4}$ Department of Home Economics, St. Angela's College, Sligo (National University of Ireland Galway), Sligo, Ireland. ${ }^{5}$ Centre for Evidence and Social Innovation, Queen's University Belfast, Belfast, UK. ${ }^{6}$ Centre for Public Health, Queen's University Belfast, Belfast, UK.

Received: 10 August 2020 Accepted: 18 January 2021

Published online: 30 January 2021

\section{References}

1. Wirt A, Collins CE. Diet quality-what is it and does it matter? Public Health Nutr. 2009;12(12):2473-92.

2. Florence MD, Asbridge M, Veugelers PJ. Diet quality and academic performance. J Sch Health. 2008;78(4):209-15.

3. Pandita A, Sharma D, Pandita D, Pawar S, Tariq M, Kaul A. Childhood obesity: prevention is better than cure. Diab Metab Synd Obes. 2016;9:83.

4. Sahoo K, Sahoo B, Choudhury AK, Sofi NY, Kumar R, Bhadoria AS. Childhood obesity: causes and consequences. J Fam Med Prim care. 2015;4(2):187.

5. Pérez-Rodrigo C, Aranceta J. School-based nutrition education: lessons learned and new perspectives. Public Health Nutr. 2001;4(1a):131-9.

6. World Health Organization. Report of the Commission on Ending Childhood Obesity. Geneva: WHO, 2016. Available online: http://www. who.int/end-childhood-obesity/ publications/echo-report/en/. Accessed 17 December 2019

7. Utter J, Fay AP, Denny S. Child and youth cooking programs: more than good nutrition? J Hunger Environ Nutr. 2017;12(4):554-80.

8. Hersch D, Perdue L, Ambroz T, Boucher JL. Peer reviewed: the impact of cooking classes on food-related preferences, attitudes, and behaviors of school-aged children: a systematic review of the evidence, 2003-2014. Prev Chronic Dis. 2014:11

9. Lavelle F, Spence M, Hollywood L, McGowan L, Surgenor D, McCloat A, Mooney E, Caraher M, Raats M, Dean M. Learning cooking skills at different ages: a cross-sectional study. Int J Behav Nutr Phys Act. 2016;13(1):1-1.

10. Laska MN, Larson NI, Neumark-Sztainer D, Story M. Does involvement in food preparation track from adolescence to young adulthood and is it associated with better dietary quality? Findings from a 10-year longitudinal study. Public Health Nutr. 2012;15(7):1150-8.

11. Mills S, Brown H, Wrieden W, White M, Adams J. Frequency of eating home cooked meals and potential benefits for diet and health: cross-sectional analysis of a population-based cohort study. Int J Behav Nutr Phys Act. 2017;14(1):109.

12. Burrows TL, Martin RJ, Collins CE. A systematic review of the validity of dietary assessment methods in children when compared with the method of doubly labeled water. J Am Diet Assoc. 2010;110(10):1501-10.

13. Burrows T, Golley RK, Khambalia A, McNaughton SA, Magarey A, Rosenkranz RR, Alllman-Farinelli M, Rangan AM, Truby H, Collins C. The quality of dietary 
intake methodology and reporting in child and adolescent obesity intervention trials: a systematic review. Obes Rev. 2012;13(12):1125-38.

14. Doustmohammadian A, Omidvar N, Keshavarz-Mohammadi N, Abdollahi M, Amini $\mathrm{M}$, Eini-Zinab $\mathrm{H}$. Developing and validating a scale to measure food and nutrition literacy (FNLIT) in elementary school children in Iran. PLoS One. 2017;12(6):e0179196.

15. Reynolds JS, Treu JA, Njike V, Walker J, Smith E, Katz CS, Katz DL. The validation of a food label literacy questionnaire for elementary school children. J Nutr Educ Behav. 2012;44(3):262-6.

16. Musher-Eizenman D, Holub S. Comprehensive feeding practices questionnaire: validation of a new measure of parental feeding practices. J Pediatr Psychol. 2007;32(8):960-72.

17. Lohse B, Cunningham-Sabo L, Walters LM, Stacey JE. Valid and reliable measures of cognitive behaviors toward fruits and vegetables for children aged 9 to 11 years. J Nutr Educ Behav. 2011;43(1):42-9.

18. Bandura A. Social foundations of thought and action. NJ: Englewood Cliffs; 1986. p. 23-8.

19. Lavelle F, Hollywood L, Caraher M, McGowan L, Spence M, Surgenor D, McCloat A, Mooney E, Raats M, Dean M. Increasing intention to cook from basic ingredients: a randomised controlled study. Appetite. 2017;116:502-10.

20. Garcia AL, Reardon R, McDonald M, Vargas-Garcia EJ. Community interventions to improve cooking skills and their effects on confidence and eating behaviour. Curr Nutr Rep. 2016;5(4):315-22.

21. Dixon EJ, Condrasky MD, Sharp JL, Corr AQ. Cooking confidence and healthy eating choices of preadolescent participants at a cooking camp. Top Clin Nutr. 2013;28(1):21-33.

22. Wrieden WL, Anderson AS, Longbottom PJ, Valentine K, Stead M, Caraher M, Lang T, Gray B, Dowler E. The impact of a community-based food skills intervention on cooking confidence, food preparation methods and dietary choices-an exploratory trial. Public Health Nutr. 2007;10(2):203-11.

23. Caraher M, Seeley A, Wu M, Lloyd S. When chefs adopt a school? An evaluation of a cooking intervention in English primary schools. Appetite. 2013;62:50-9.

24. Losier GF, Vallerand RJ. The temporal relationship between perceived competence and self-determined motivation. J Soc Psychol. 1994;134(6): 793-801.

25. Harter S. Effectance motivation reconsidered. toward a developmental model. Hum Dev. 1978:21(1):34-64.

26. Crocker PR, Eklund RC, Kowalski KC. Children's physical activity and physical self-perceptions. J Sports Sci. 2000;18(6):383-94.

27. Davison KK, Downs DS, Birch LL. Pathways linking perceived athletic competence and parental support at age 9 years to girls' physical activity at age 11 years. Res Q Exerc Sport. 2006;77(1):23-31.

28. Bardid F, De Meester A, Tallir I, Cardon G, Lenoir M, Haerens L. Configurations of actual and perceived motor competence among children: associations with motivation for sports and global self-worth. Hum Mov Sci. 2016:50:1-9.

29. Barnett LM, Ridgers ND, Salmon J. Associations between young children's perceived and actual ball skill competence and physical activity. J Sci Med Sport. 2015;18(2):167-71

30. Barnett LM, Vazou S, Abbott G, Bowe SJ, Robinson LE, Ridgers ND, Salmon J, Construct validity of the pictorial scale of perceived movement skill competence. Psychol Sport Exerc. 2016;22:294-302

31. Punch $\mathrm{S}$. Research with children: the same or different from research with adults? Childhood. 2002:9(3):321-41.

32. Boyden J, Ennew J. Children in focus: a manual for experiential learning in participatory research with children. Stockholm: Rädda Barnen; 1997.

33. Mann G, Tolfree D. Children's participation in research: reflections from the care and protection of separated children in emergencies project. Stockholm: Save the Children Sweden; 2003.

34. Morgan PJ, Young MD, Barnes AT, Eather N, Pollock ER, Lubans DR. Engaging fathers to increase physical activity in girls: the "dads and daughters exercising and empowered"(DADEE) randomized controlled trial. Ann Behav Med. 2019;53(1):39-52.

35. Brian A, Bardid F, Barnett LM, Deconinck FJ, Lenoir M, Goodway JD. Actual and perceived motor competence levels of Belgian and United States preschool children. J Motor Learn Dev. 2018;6(s2):S320-36.

36. Estevan I, Molina-García J, Abbott G, Bowe SJ, Castillo I, Barnett LM. Evidence of reliability and validity for the pictorial scale of perceived movement skill competence in Spanish children. J Motor Learn Dev. 2018; 6(s2):S205-22.
37. Farmer $\mathrm{O}$, Belton $\mathrm{S}, \mathrm{O}$ 'Brien $\mathrm{W}$. The relationship between actual fundamenta motor skill proficiency, perceived motor skill confidence and competence, and physical activity in 8-12-year-old Irish female youth. Sports. 2017;5(4):74

38. Peers C, Issartel J, Behan S, O'Connor N, Belton S. Movement competence: association with physical self-efficacy and physical activity. Hum Mov Sci. 2020;70:102582.

39. Dean M, O'Kane C, Issartel J, McCloat A, Mooney E, Gaul D, Wolfson J, Lavelle F. Guidelines for designing age-appropriate cooking interventions for children: the development of evidence-based cooking skill recommendations for children, using a multidisciplinary approach. Appetite, 2021. https://doi.org/10.1016/j.appet.2021.105125.

40. Lavelle F, McGowan L, Hollywood L, Surgenor D, McCloat A, Mooney E, Caraher M, Raats M, Dean M. The development and validation of measures to assess cooking skills and food skills. Int J Behav Nutr Phys Act. 2017;14(1):1-3.

41. Horne PJ, Tapper K, Lowe CF, Hardman CA, Jackson MC, Woolner J. Increasing children's fruit and vegetable consumption: a peer-modelling and rewards-based intervention. Eur J Clin Nutr. 2004:58(12):1649-60.

42. Lowe CF, Horne PJ, Tapper K, Bowdery M, Egerton C. Effects of a peer modelling and rewards-based intervention to increase fruit and vegetable consumption in children. Eur J Clin Nutr. 2004;58(3):510-22

43. Kirby P. Involving young researchers: how to enable young people to design and conduct research. York: York Publishing Services; 1999.

44. Brennan SF, Lavelle F, Moore SE, Dean M, McKinley MC, McCole P, Hunter RF, Dunne L, O'Connell NE, Cardwell CR, Elliott CT, McCarthy D, Woodside $J V$. Food environment intervention improves food knowledge, wellbeing and dietary habits in primary school children: project Daire, a randomisedcontrolled, factorial design cluster trial. IJBNPA, 2021. https://doi.org/10. 1186/s12966-021-01086-y.

45. Yong AG, Pearce $S$. A beginner's guide to factor analysis: focusing on exploratory factor analysis. Tutor Quan Methods Psychol. 2013;9(2):79-94.

46. Kaiser HF. An index of factorial simplicity. Psychometrika. 1974;39(1):31-6.

47. Field A. Discovering statistics using IBM SPSS statistics. sage; 2013.

48. Kaiser HF. The application of electronic computers to factor analysis. Educ Psychol Meas. 1960;20(1):141-51.

49. Carpenter $\mathrm{S}$. Ten steps in scale development and reporting: a guide for researchers. Commun Methods Meas. 2018;12(1):25-44.

50. Hinkin TR, Tracey JB, Enz CA. Scale construction: developing reliable and valid measurement instruments. J Hosp Tourism Res. 1997;21(1):100-20.

51. Hair JF, Black WC, Babin BJ, Anderson RE, Tatham RL. Multivariate data analysis. Upper Saddle River, NJ: Prentice hall; 1998.

52. Koo TK, Li MY. A guideline of selecting and reporting intraclass correlation coefficients for reliability research. J Chiropract Med. 2016;15(2):155-63.

53. Hays RD, Hadorn D. Responsiveness to change: an aspect of validity, not a separate dimension. Qual Life Res. 1992;1(1):73-5.

54. Bland JM, Altman DG. Statistics notes: Cronbach's alpha. BMJ. 1997; 314(7080):572

55. Rattray J, Jones MC. Essential elements of questionnaire design and development. J Clin Nurs. 2007:16(2):234-43.

56. Lavelle F, Benson T, Hollywood L, Surgenor D, McCloat A, Mooney E, Caraher M, Dean M. Modern transference of domestic cooking skills. Nutrients. 2019;11(4):870

57. Ronto $\mathrm{R}$, Ball $\mathrm{L}$, Pendergast $\mathrm{D}$, Harris ND. Food literacy at secondary schools in Australia. J Sch Health. 2016;86(11):823-31.

58. Asher RC, Jakstas T, Wolfson JA, Rose AJ, Bucher T, Lavelle F, Dean M, Duncanson K, Innes B, Burrows T, Collins CE. Cook-EdTM: a model for planning, implementing and evaluating cooking programs to improve diet and health. Nutrients. 2020;12(7):2011.

\section{Publisher's Note}

Springer Nature remains neutral with regard to jurisdictional claims in published maps and institutional affiliations. 